\title{
Symmetry breakage in the development of one-armed gonads in nematodes
}

\author{
Marie-Anne Félix and Paul W. Sternberg \\ Howard Hughes Medical Institute, Division of Biology, 156-29, Caltech, Pasadena CA 91125, USA
}

\section{SUMMARY}

Whereas the hermaphrodite gonad of Caenorhabditis elegans has two symmetric arms (didelphy), the female/hermaphrodite gonad of many nematode species features a single anterior arm (monodelphy). We examined how gonadal cell lineages and intercellular signalling evolve to generate these diverse structures. In C. elegans, the two arms develop symmetrically from two somatic precursor cells, $\mathrm{Z1}$ (anterior) and $\mathrm{Z4}$ (posterior). Each first gives rise to one distal tip cell (which promotes arm growth and germ line proliferation), two ovary precursors and three uterine precursors in the center of the developing gonad. In monodelphic species, $\mathrm{Z1}$ and $\mathrm{Z4}$ have different fates. The first visible asymmetry between them is in the relative timing of their divisions, followed by asymmetric cell movements. The putative posterior distal tip cell is then eliminated in all but one species by programmed cell death. In some species the posterior ovary precursors form a small vestigial posterior arm, the post-vulval sac; in other species, they stay undivided, or die. In Cephalobus sp. PS1197, the specific fate of $\mathrm{Z4}$ progeny is induced by $\mathrm{Z1}$ (or its daughters). In the uterus in $C$. elegans, symmetric lateral signalling between $Z 1$.ppp and $Z$ 4.aaa renders them equally likely to become the anchor cell, which links the uterus to the vulva. In the different monodelphic species, anchor cell specification is biased, or fully fixed, to a descendant of either $\mathrm{Z1}$ or $\mathrm{Z4}$. Replacement regulation upon anchor cell ablation is conserved in some species, but lost in others, leading to a mosaic-type development. Differentiation between $\mathrm{Z1}$ and $\mathrm{Z4}$ is thus manifested at this later stage in the breakage of symmetry of cell interactions in the ventral uterus.

Key words: gonad, evolution, nematodes, cell lineage, apoptosis, lateral signalling

\section{INTRODUCTION}

Biological complexity evolves in part through processes of diversification, in which a symmetry is broken and new, distinct, asymmetric features appear. This holds true for the differentiation of segments and thagma in arthropods (Akam et al., 1994; Carroll, 1994), or for the asymmetric differentiation of flower organs (Coen and Nugent, 1994). Another dramatic example is the breakage of symmetry in the female/hermaphrodite gonad of nematode worms. We study it as an example of evolutionary diversification.

Many nematode species, like Caenorhabditis elegans, develop two gonadal arms related by rotational symmetry. The two arms are built symmetrically from two somatic precursor cells, Z1 and Z4 (Kimble and Hirsh, 1979): Z1 gives rise to the anterior-right arm, $\mathrm{Z} 4$ to the posterior-left arm, and both contribute to the common central uterus. Symmetry of the organ is thus reflected at the cellular level in the symmetry of these cells and their progeny. A single arm develops in a number of other nematodes; in the species of the order Rhabditida considered here, the single arm is always anterior; a variably developed structure, called the post-vulval sac, grows posterior to the uterus in some of these species. In the monodelphic Panagrellus redivivus, $\mathrm{Z} 1$ gives rise to the anterior arm; $\mathrm{Z} 4$ has a novel fate and gives rise to the post-vulval sac, but also contributes to the uterus (Sternberg and Horvitz, 1981). Asymmetry of the gonad is thus reflected at the cellular level in the distinct fates of $\mathrm{Z} 1$ and $\mathrm{Z} 4$.

Many free-living nematodes, like Caenorhabditis elegans, are easy to culture, observe and manipulate, permitting not only a precise description, but also a mechanistic analysis of their development by cell ablation and genetics (Sommer et al., 1994; Sommer and Sternberg, 1996). Therefore we believe that the development of one-armed gonads in nematodes is a good system to study evolutionary diversification. Here we present the cellular description of asymmetric gonad development in an array of species from distinct evolutionary lines, and we examine the cell interactions involved in breaking symmetries between the $\mathrm{Z} 1$ and $\mathrm{Z} 4$ lineages.

\section{MATERIALS AND METHODS}

Oscheius guentheri Sudhaus (Sudhaus and Hooper, 1994) was kindly provided by W. Sudhaus, University of Berlin. All other strains were provided by L. Carta and are designated by their strain number in the Caltech collection: Oscheius sp. (closely related to O. tipulae; L. Carta, K. Thomas and P. W. S., unpublished data) (PS1131), collected in Tokyo, Japan, in July 1991 by W. Wood; Acrobeloides sp. $c f$. amurensis (Truskova, 1971) (PS1146), collected in Blythe, California in February 1992 by R. Wellman; Cephalobus cubaensis (Steiner, 1935) (PS1197), collected in Nadi, Fiji Islands by W. Boorstein; Panagrolaimus sp. (PS1579), collected in the Huntington Gardens, San Marino, California in July 1994 by M.-A. F. and P. W. S.; Pana- 
grolaimus sp. (PS1732), collected near Iceberg Lake, California in July 1994 by J. Demodena; Mesorhabditis sp. (PS1179), collected in Mira Loma, California in September 1992. PS1146 and PS1197 were identified by P. De Ley, Gent.

For strain culture, cell lineage and laser ablation, we used standard techniques described for Caenorhabditis elegans (Epstein and Shakes, 1995; Wood, 1988). Worms were kept at $20-25^{\circ} \mathrm{C}$. Gonad lineages were observed at $20^{\circ} \mathrm{C}$. Lineages are based on at least three animals, followed continuously by Nomarski optics for the period in between each round of divisions, and partial observations on more than 20 individuals. The life cycle of Oscheius guentheri is approximately 6.5-7 days long at $20^{\circ} \mathrm{C}, 3$ days at $25^{\circ} \mathrm{C}$ and 2 days at $30^{\circ} \mathrm{C}$; of Acrobeloides $s p$. PS1 14615 , days at $20^{\circ} \mathrm{C}$ and 10 days at $25^{\circ} \mathrm{C}$; of Cephalobus $s p$. PS1197, $19-20$ days at $20^{\circ} \mathrm{C}, 7$ days at $25^{\circ} \mathrm{C}$ and 4 days at $30^{\circ} \mathrm{C}$; of Panagrolaimus sp. PS1579, 9 days at $20^{\circ} \mathrm{C}$ and 6 days at $25^{\circ} \mathrm{C}$; of PS1732, 10 days at $20^{\circ} \mathrm{C}, 5-6$ days at $25^{\circ} \mathrm{C}$ and $3.5-4$ days at $30^{\circ} \mathrm{C}$; of Mesorhabditis sp. PS1179, about 7 days at $20^{\circ} \mathrm{C}$. Oscheius species, Acrobeloides sp. PS1146 and Panagrolaimus sp. PS1732 are hermaphroditic. Cephalobus cubaensis PS1197 and Panagrolaimus sp. PS1579 are parthenogenetic. Mesorhabditis sp. PS1179 is a malefemale species.

\section{RESULTS}

\section{Symmetric and asymmetric gonads}

A major morphological change among nematode species concerns the number of arms of the female/hermaphrodite gonad (Chitwood and Chitwood, 1974). This character varies in the order Rhabditida between one (monodelphy) and two (didelphy) (Lorenzen, 1981). For the set of species studied here (all of the order Rhabditida), it is possible to infer the phylogeny presented in Fig. 1, based on morphological data (Andrássy, 1976, 1984; Maggenti, 1981; Sudhaus, 1976; Sudhaus and Hooper, 1994) (molecular phylogenetic analysis for these species is underway; K. Thomas, personal

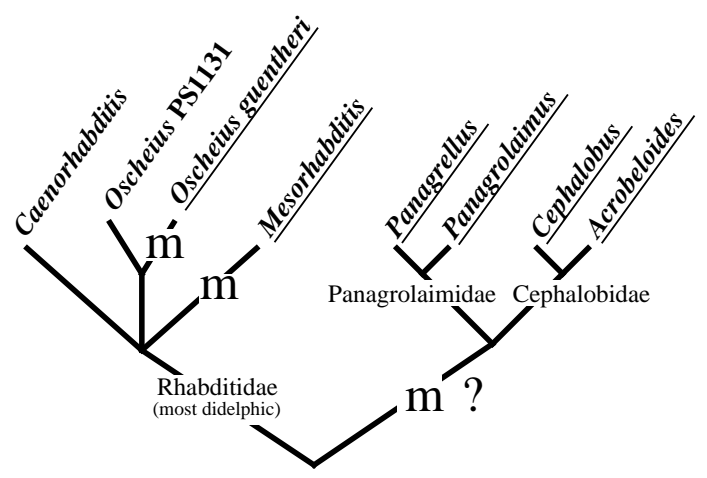

Fig. 1. Proposed phylogenetic relationships between the species used in this study. The phylogeny shown is based on morphological data (Andrássy, 1976, 1984; Maggenti, 1981; Sudhaus, 1976; Sudhaus and Hooper, 1994). Branch lengths do not reflect phylogenetic distances. Monodelphic genera/species are underlined, 'm' marks the acquisition of monodelphy in the branch. The set of species comprises representatives of the three families Rhabditidae, Panagrolaimidae and Cephalobidae (order Rhabditida). The family Rhabditidae has a majority of didelphic species (not listed here), and monodelphy was acquired independently for Mesorhabditis spp. and Oscheius guentheri. The two other families are entirely

monodelphic, and it is not clear whether their monodelphy is derived or primitive relative to didelphy in the family Rhabditidae.

Relationships among the three families are also unclear. communication). It is not clear whether the common nematode ancestor had a one-armed or a two-armed gonad (Lorenzen, 1981), and how often the character changed in a given line. Nonetheless, monodelphy is clearly derived for Oscheius guentheri relative to the other species of this genus (Sudhaus and Hooper, 1994). This phylogeny suggests not only that monodelphy may have independently evolved several times, but also that it is a distinctive character of taxa of different sizes: Panagrolaimidae and Cephalobidae are entire monodelphic families, Mesorhabditis is a monodelphic genus (or subgenus in the nomenclature of Sudhaus, 1976), distantly related to Caenorhabditis and other didelphic genera of the family Rhabditidae. Oscheius guentheri is a single monodelphic species in an otherwise didelphic genus (Sudhaus and Hooper, 1994). This last case is particularly interesting as it appears to be a recent evolutionary event of reduction of the posterior arm.

Monodelphic gonads can have a variety of morphologies (see the schematic drawings in Fig. 2). In the species considered here, the single arm is always anterior. It elongates from the gonad primordium in the center of the animal first anteriorly, then performs a dorsal turn and folds back posteriorly. In Oscheius guentheri, the anterior arm does not elongate more than in a didelphic species (Sudhaus and Hooper, 1994). By contrast, in the other monodelphic species we studied, the anterior arm extends far into the posterior part of the animal. In $O$. guentheri, the posterior arm always contains some germ line. It has a variable size, from a relatively short post-vulval sac (Fig. 3B) to a sterile posterior arm of almost normal size (Fig. 3C). In Acrobeloides sp. PS1146, the post-vulval sac is relatively long and often contains some germ line (Fig. 3E, F). In Panagrolaimus spp. and Cephalobus sp. PS1197, the postvulval sac is shorter and does not contain any germ line. In Mesorhabditis sp., the post-vulval sac is not present and the vulva is shifted posteriorly, relative to the other species considered, in which it is approximately central.

\section{Review of the symmetric development of Z1 and Z4 in Caenorhabditis elegans}

The two-armed gonad of $C$. elegans hermaphrodites is built symmetrically during post-embryonic development from two somatic precursors, Z1 and Z4, and two germ line precursors Z2 and Z3 (Kimble and Hirsh, 1979). During the first larval stage (L1), Z1 and Z4 first each generate six descendants (Fig. 2): distally, three ovary precursors (dark and light blue), and centrally, three uterine precursors (yellow and orange). Divisions then pause, and these twelve cells interact and rearrange their positions to form the 'somatic primordium' at the end of L2. The cells are named according to Sulston and Horvitz (1977): the anterior and posterior daughters of Z1 are Z1.a and Z1.p, respectively; the daughters of Z1.a are Z1.aa and Z1.ap, etc.

Z1.aa and Z4.pp are the most distal cells of each arm (distal tip cells or DTC, dark blue). They lead arm growth and germ line proliferation (Kimble and White, 1981). Two ovary precursors in each arm (light blue) further divide and differentiate. The uterus is generated in the center from three $\mathrm{Z} 1$ and three Z4 descendants: Z1.pap and Z4.apa generate predominantly the dorsal uterus, and the four daughters of Z1.pp and $\mathrm{Z} 4$.aa the ventral uterus. One of the latter becomes the anchor cell (AC, orange) which links the uterus to the vulva, and does 

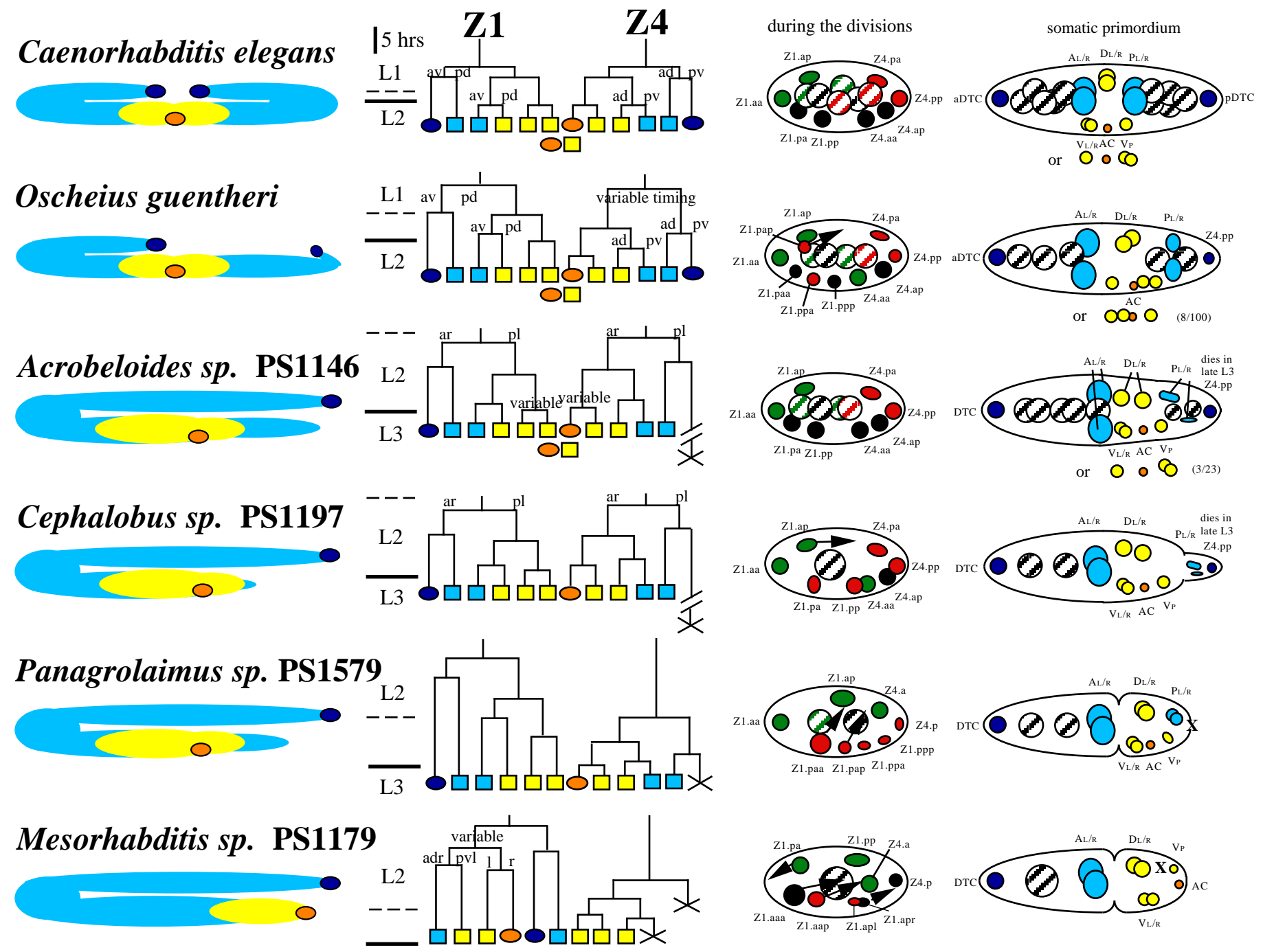

Fig. 2. Early gonad development in didelphic and monodelphic species. (Left) Morphology of the gonad, lateral view. Blue, ovary; yellow, uterus; dark blue oval, distal tip cell (DTC); orange oval, anchor cell (AC). Anterior is to the left, dorsal to the top throughout the figure. (Center) Early cell lineages of the somatic gonad precursor cells Z1 and Z4, and the general fates of their progeny. Light blue squares, ovary precursors; yellow squares, uterus; X, cell death. The branches of the lineage are oriented anterior to the left, unless otherwise specified (v, ventral; d, dorsal; p, posterior; a, anterior; 1 left; $r$, right). The timing relative to the larval stages is shown on the left of the lineage diagrams. The dashed lines indicate entry into the lethargic stage preceding each molt. (Right) Schematic positions of the nuclei during gonad development. The first stage displays the position of the nuclei at some point during the early gonad divisions. Since the relative timing of $Z 1$ and Z4 development varies between species, the different diagrams at this stage are in different stages of development for Z1 and Z4. Cells are named according to lineage history and colored according to their left-right position (red, left; black, medial; green, right). In the somatic primordium stage, they are labeled and colored according to their fate (following the color codes shown in the lineage). Nomenclature follows that of Sternberg and Horvitz (1981). Germ line precursors are hatched. At hatching, there are two germ line precursors (Z2 and Z3) in all species except PS1197 and PS1179, which have a single one ('Z23'). The timing of their division is somewhat variable from animal to animal, and varies extensively between species.

not divide further. In C. elegans, the anchor cell is Z1.ppp in $50 \%$ of the individuals, Z4.aaa in the others. Interactions between Z1.ppp and Z4.aaa determine which becomes the AC and which becomes the VU (Kimble, 1981; Seydoux and Greenwald, 1989). If one cell is ablated in L2, the other one always becomes an AC.

In summary, symmetric, two-armed gonads of female/hermaphrodite nematodes are formed through the symmetric development of the two somatic precursor cells, Z1 and Z4 (Chin, 1977; Chitwood and Chitwood, 1974; Hirschmann and
Triantaphyllou, 1967). This holds true not only in Caenorhabditis elegans, but also in Oscheius sp. PS1131 and Pristionchus pacificus (Sommer et al., 1996; family Diplogastridae), two other didelphic species (M.-A. F., unpublished). Z1 and Z4 have symmetric fates: each of the two distal tip cells is responsible for the growth of an arm; in the center, where cells from both Z1 and Z4 lineages interact, intercellular signalling determines which of the ventral uterine precursors becomes the single anchor cell. We describe successively these different aspects in the development of one-armed gonads. 


\section{Asymmetric development of $\mathrm{Z1}$ and $\mathrm{Z4}$ in monodelphic species}

We observed the early development of the female/hermaphrodite gonad of five monodelphic species (Fig. 2). The conservation of gonad development between the different (didelphic and monodelphic) species is remarkable and forms the basis for comparative studies at the level of the individual progeny of $\mathrm{Z} 1$ and $\mathrm{Z} 4$. The gonad always develops from two somatic precursor cells that divide in a characteristic pattern and produce specific blast cells (Fig. 2) (Chitwood and Chitwood, 1974). A period of cell rearrangements follows these early divisions and leads to the 'somatic primordium' stage when cells adopt a very characteristic, invariant configuration. In didelphic species, all cells of the somatic gonad are in the center, except the two distal tip cells, which migrate out followed by the proliferating germ line (Kimble and Hirsh, 1979). In the monodelphic species we studied, all cells except the anterior distal tip cell are clustered at the posterior end of the primordium (Fig. 2) (the converse is true in males, where most cells cluster anteriorly; Kimble and Hirsh, 1979; Sternberg and Horvitz, 1981). This observation extends to monodelphic species with a single anterior arm in other orders of nematodes (Anderson and Darling, 1964; Hirschmann, 1962, 1977; Roman and Hirschmann, 1969). The timing of the early gonad divisions varies extensively from species to species relative to the larval stages (Fig. 2), but the developmental period after the early divisions is probably constrained by the cell determinations and interactions that take place, before the cells start dividing again to form the distinct parts of the gonad. It is an obligate 'organotypic' period in gonad development, analogous (at a smaller scale) to the phylotypic period in embryonic development (Duboule, 1994; Slack et al., 1993).

Early gonad development in Panagrolaimus sp. PS1579 closely resembles that of Panagrellus redivivus (Sternberg and Horvitz, 1981) (except for some timing differences). However, we find variations in the development of monodelphic gonads in species that are more distant phylogenetically.

In all cases, the first visible asymmetry between the Z1 and $\mathrm{Z} 4$ lineages is the relative timing of their divisions (sometimes preceded by the larger size of the nucleus of the cell that will divide first). In Oscheius guentheri, the relative timing of $\mathrm{Z} 1$ and $\mathrm{Z} 4$ divisions is variable from individual to individual, but Z4 usually divides after Z1 (20/25 animals). Later divisions of the Z4 lineage are still further delayed. By contrast, in the didelphic Oscheius PS1131, there is no apparent bias as to which cell divides first (Z4 divided first in 11/21 animals). All other monodelphic species studied show a clear asymmetry in the timing of $\mathrm{Z} 1$ division relative to $\mathrm{Z} 4$ division: $\mathrm{Z} 4$ reproducibly divides 1 hour before $\mathrm{Z} 1$ in Acrobeloides and Cephalobus spp., and about 12 hours after in Panagrolaimus and Mesorhabditis spp. This lag suggests that $\mathrm{Z} 1$ and $\mathrm{Z} 4$ are different at, or prior to, their first division.

In Oscheius guentheri, the position of the nuclei of $\mathrm{Z} 1$ and Z4 progeny is symmetric in early L2 (Fig. 2). This spatial symmetry is then broken, seemingly through the differential activities of Z1.aa and Z4.pp; the latter (the presumptive distal tip cell on the posterior side) induces less proliferation in the germ line on its side, migrates less rapidly and stays closer to the uterine precursors (Fig. 3A). The development of the entire posterior part of the gonad is delayed; ultimately there are signs of partial germ line differentiation in the posterior arm in old adults (see the drawings in Sudhaus and Hooper, 1994). In intact animals, the part of the gonad posterior to the uterus is at least as long as one body width, and always contains more than one germ line precursor in the L4 (10/10 animals). After ablation of Z4.pp in young L2 larvae, the part of the gonad posterior to the uterus stayed shorter than one body width
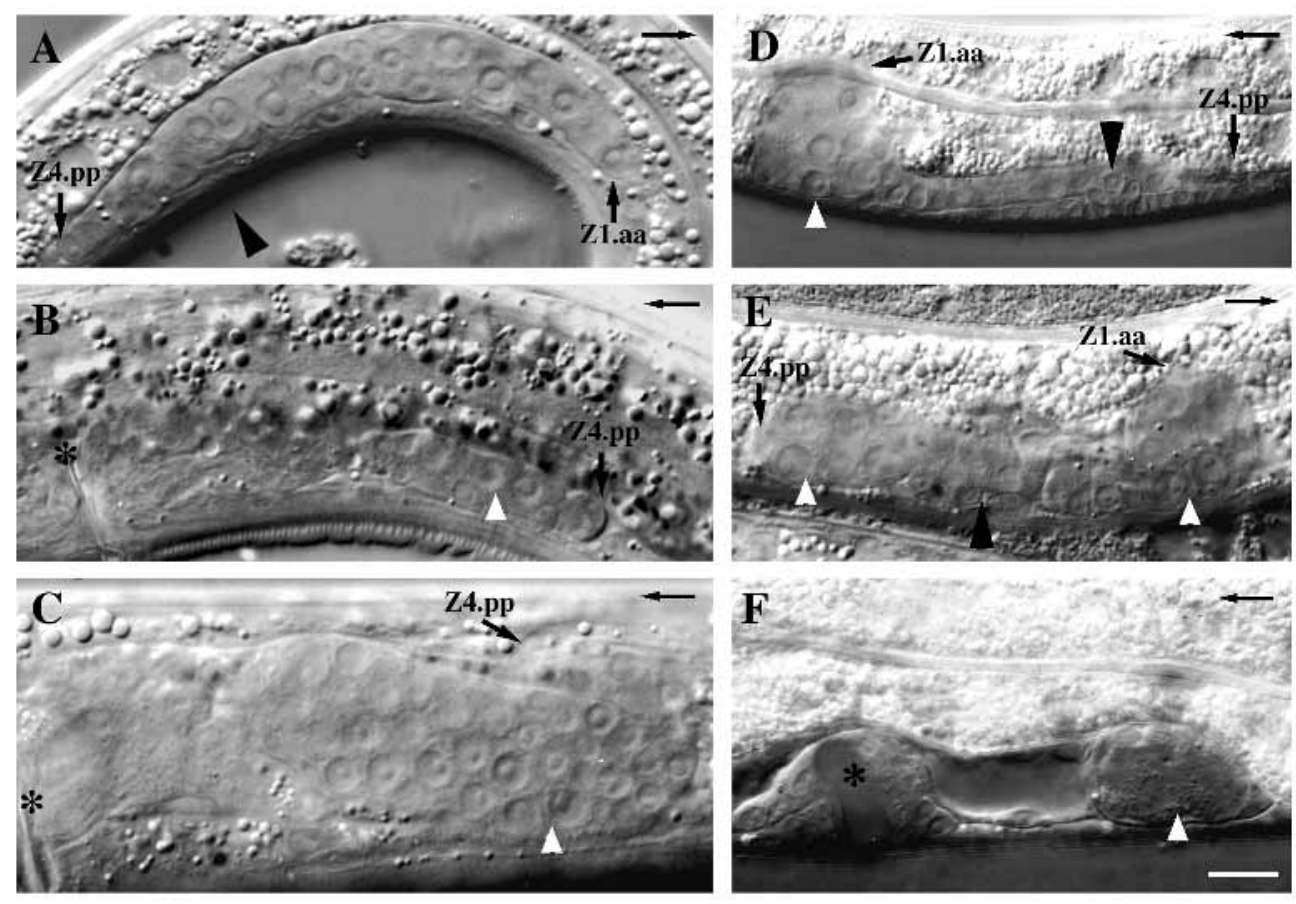

Fig. 3. Variable asymmetric development of the posterior arm in Oscheius guentheri and Acrobeloides sp. PS1146.

(A) Oscheius guentheri, early L3. (B,C) Young adults. (D,E) Acrobeloides sp. PS1146, late L3. (F) Late L4. Lateral views in Nomarski optics; the arrow in the upper right corner of each part points towards the head, dorsal side is at the top. Black arrowheads designate the antero-posterior position of the anchor cell (only visible in the plane of focus in D). Asterisks indicate the vulva. Bar: $10 \mu \mathrm{m}$. In these two species, gonad development stays symmetric during the early divisions of $\mathrm{Z1}$ and Z4. Then Z1.aa is more active than Z4.pp in promoting arm growth $(\mathrm{A}, \mathrm{D}, \mathrm{E})$. The development of the posterior arm is variable within a species: germ line precursors (large nuclei, white

arrowheads) proliferated much more in C than in B and are found only in the anterior arm in D, but in both arms in E. F shows a post-vulval sac filled with germ line; the uterus lumen extends over the vulval invagination. 

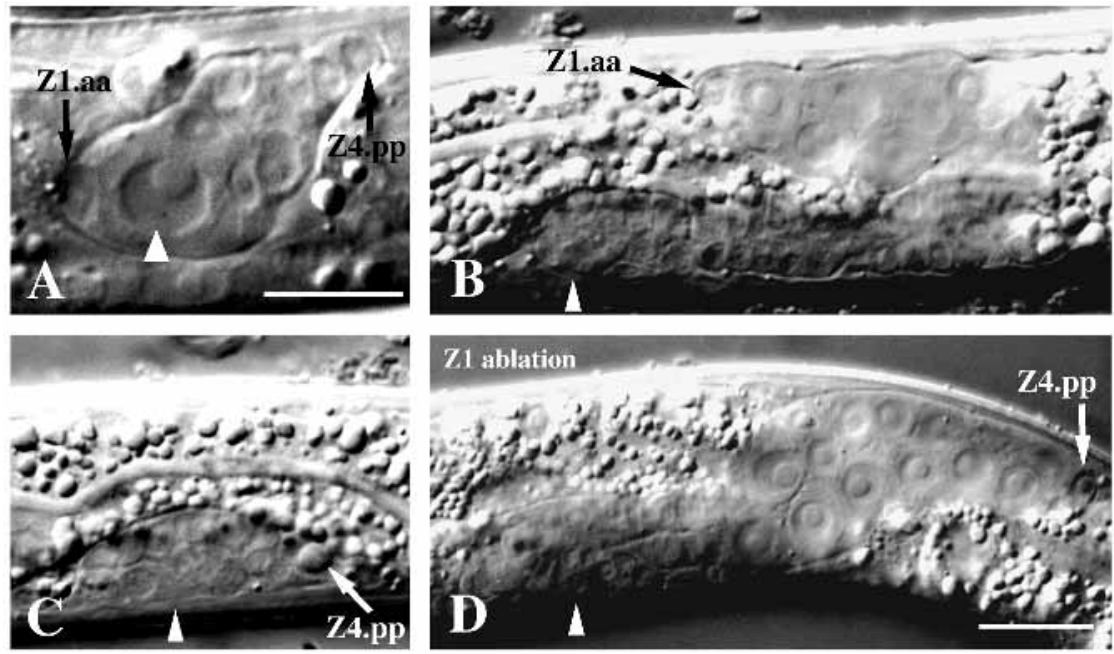

Fig. 4. Gonad development in Cephalobus sp. PS1197. (A) Ventral view of the gonad primordium in late L2. Anterior is to the left. $\mathrm{Z} 1$.aa is the only cell anterior to the germ line precursor (large nucleus, white arrowhead). (B) Anterior gonadal arm in late L3: germ line precursors (large nuclei) proliferate next to Z1.aa. Arrowheads in B-D designate the position of the vulva. (C) Underdeveloped posterior arm in late L3: Z4.pp is dying (refractile appearance).

(D) Growth of a posterior arm after ablation of $\mathrm{Z} 1$ in early L1: the germ line proliferated next to Z4.pp, which survived (early L4). B-D are lateral views, anterior is to the right in $\mathrm{B}$, to the left in $\mathrm{C}$, D. Bar: $10 \mu \mathrm{m}$; same scale in B-D.
(11/11 animals, scored in L4), and did not contain any germ line in 6/11 animals; a single undivided germ line precursor was found in the remaining 5/11 animals. Z4.pp thus retains some activity as a distal tip cell, but appears less active than its anterior counterpart, Z1.aa.

In Acrobeloides sp. PS1146, spatial symmetry also appears to be broken after the early gonad divisions are completed, through the differential activity of Z1.aa and Z4.pp. In the three other species, spatial asymmetry arises earlier when all Z1 progeny (except Z1.aa) migrate towards the Z4 descendants, leaving all of the germ line next to the anterior distal tip cell. The somatic gonad primordium is then very asymmetric (Fig. 2). In Cephalobus sp. PS1197 and Mesorhabditis sp. PS1179, there is a single germ line precursor at hatching (Z23). It divides late in gonad development, by which time it is asymmetrically positioned in the gonad primordium (Figs 2, 4A, 5A).

\section{Cell death in the posterior ovary}

Differential activity of the anterior and posterior distal tip cells (Z1.aa and Z4.pp respectively) is thus important in shaping the gonad in some monodelphic species. Strikingly, in all species except Oscheius guentheri, Z4.pp is eliminated by programmed cell death (as revealed by its refractile aspect in Nomarski optics), thereby removing this putative distal tip cell (Fig. 2). Its death occurs shortly after its birth in Panagrolaimus sp. PS1579 as in Panagrellus redivivus, thus eliminating its function altogether (Sternberg and Horvitz, 1981). It dies one larval stage later (at the molt to the fourth larval stage) in Acrobeloides sp. PS1146 and Cephalobus sp. PS1197 (Fig. 4C).

The posterior ovary precursors generate the post-vulval sac in Panagrellus redivivus (Sternberg and Horvitz, 1981), Panagrolaimus sp. PS1579 and Acrobeloides sp. PS1146. They divide later than their anterior counterparts, and variably. In Cephalobus PS1197, which has a short post-vulval sac (developmentally, the posterior part of the uterus), they do not divide. In Mesorhabditis, which has a posterior vulva and no postvulval sac, Z4.p and Z4.app die, eliminating the distal tip cell and the two presumptive ovary precursors (Fig. 2). Thus the program of the posterior ovary precursors is modified to fewer divisions or even cell death. Together with the decreased activity or the death of the presumptive posterior distal tip cell, these cell fate changes cause reduction of the posterior arm.

\section{Z1 induces the Z4 fate in Cephalobus sp. PS1197}

As shown above, Z1 and Z4 are different in monodelphic species before they divide. We studied possible interactions between them by ablating one and observing the development of the other. Ablation of Z1 prevents Z4.pp death and causes growth of a posterior arm in Cephalobus sp. PS1197 (Table 1, Fig. 4D). Survival of Z4.pp is required for posterior arm growth. The arm is always sterile, but the germ line proliferates and partially differentiates into large, abnormal oocytes. Interestingly, after its dorsal turn, Z4.pp migrates posteriorly, instead of anteriorly as does the posterior distal tip cell in didelphic species of this order. (The same is observed in the Z4-ablated animals, in which the arm formed by Z1 grows posteriorly; see footnote to Table 1).

The capacity for regulation of the $\mathrm{Z} 4$ fate ends around or shortly after the time of $\mathrm{Z} 1$ and $\mathrm{Z} 4$ division, certainly before Z1.aa or Z4.pp are born (Table 1, bottom part). We conclude that in Cephalobus sp. PS1197, signalling from Z1 to Z4, or between their daughters, induces a specific fate for Z4 (in particular, the death of Z4.pp). This asymmetric signalling is an early sign of a primary asymmetry between the two cells, crucial in initiating the distinct developmental program of Z4.

By contrast, in the related Acrobeloides sp. PS1146, ablation of Z1 does not restore growth of an arm from Z4; and in Panagrolaimus spp., ablation of either of them does not modify the lineage of the other (not shown): their fates are autonomous from each other. We do not know the direction of evolution of this character between Cephalobus sp. PS1197 and Acrobeloides sp. PS1146, but this can be tested once a phylogenetic tree is established for the Cephalobidae. It could be that the distinct Z4 program was originally activated by asymmetric intercellular signalling, and that later in evolution the primary asymmetry was directly converted in specific autonomous programs for Z1 and Z4.

\section{A novel morphogenetic role for the anchor cell in Mesorhabditis sp. PS1179}

The anchor cell is a specialized cell of the uterus that connects the uterus with the vulval opening in the external epithelium 
Table 1. Z1 induces Z4 in Cephalobus sp. PS1197

\begin{tabular}{|c|c|c|c|c|c|}
\hline \multirow[b]{2}{*}{ Cell(s) ablated } & \multirow[b]{2}{*}{ Time(s) of ablation } & \multicolumn{4}{|c|}{ Gonadal arm growth* } \\
\hline & & Growth of an anterior arm & Growth of a posterior arm & No arm growth & Total \\
\hline- & - & 100 & 0 & 0 & 100 \\
\hline $\mathrm{Z4}$ & Early L1 & 11 & 7 & 2 & 20 \\
\hline $\mathrm{Z1}$ & Early L1 & 1 & 16 & 2 & 19 \\
\hline $\mathrm{Z} 1+\mathrm{Z} 23$ & Early L1 & 2 & 3 & 2 & 7 \\
\hline $\begin{array}{l}\mathrm{Z} 1 \\
+\mathrm{Z} 4 . \mathrm{pp}\end{array}$ & $\begin{array}{l}\text { Early L1 } \\
\text { Early L3 }\end{array}$ & 0 & 0 & 5 & 5 \\
\hline $\mathrm{Z1}$ & Early-mid L2 & 0 & 11 & 1 & 12 \\
\hline $\mathrm{Z1}$ & $\mathrm{Z} 4$ or $\mathrm{Z} 1$ dividing & 3 & 8 & 3 & 14 \\
\hline Z1.a + Z1.p & Early & 0 & 2 & 4 & 6 \\
\hline Z1.a + Z1.p & Late & 0 & 0 & 8 & 8 \\
\hline
\end{tabular}

*Number of animals scored in L4 with an anterior gonadal arm, or a posterior one, or none (animals with both an anterior and a posterior arm were never observed). Ablation of Z4 can disrupt the normal orientation of arm growth, presumably by inverting the axis of the first division of Z1. Ablation of Z1 allows growth of a (generally posterior) arm from the progeny of $Z 4$, even after ablation of ' $Z 23$ ', the single germ line precursor present at hatching in this species. The survival of Z4.pp upon Z1 ablation is required for arm growth. Regulation does not occur if Z1 daughters are ablated, at least late after Z1 division ('early' and 'late' are distinguished by the morphology of the nuclei of the Z1 daughters).

of the animal. In Caenorhabditis elegans, it induces the formation of the vulva (Horvitz and Sternberg, 1991; Kimble, 1981), the late differentiation of cell types in the ventral uterus (Newman et al., 1995), and continues to interact with the vulval progeny, thus helping to establish the connection

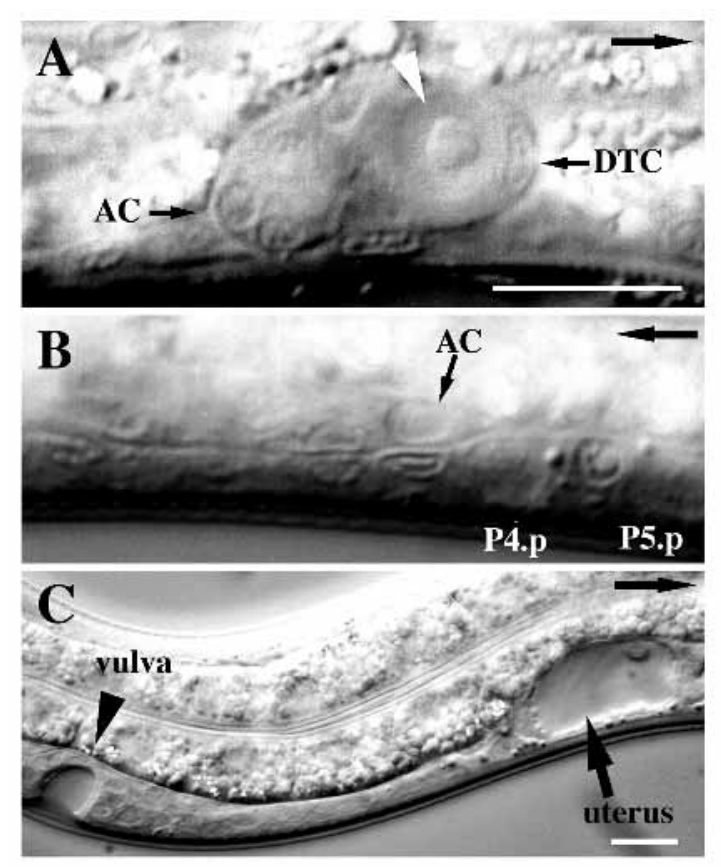

Fig. 5. The anchor cell leads the elongation of the gonad towards the posterior vulva in Mesorhabditis sp. PS1179. (A) Gonad primordium in the mid-L2 stage (between Z4 and Z4.a divisions): the future distal tip cell is the only cell anterior to the germ line precursor (white arrowhead), the future anchor cell is the most posterior cell. (B) L3: the AC migrates posteriorly towards the vulva precursor cells (P5.p to P7.p), between the intestinal cells (bright granules) and the ventral epidermis. The nuclei of the ventral uterine precursors are visible in the gonad, all anterior to the AC. (C) After AC ablation in early L3, the gonad does not elongate towards the vulval cells and the uterus forms in the center of the animal (L4 stage; compare with Fig. 2 in Sommer and Sternberg, 1994). Lateral views; the arrow in the upper right corner points towards the head, dorsal side is at the top. Bar: $10 \mu \mathrm{m}$; same scale in A-B. between the uterus and the vulva (K. Tietze and P. W. S., unpublished).

In Mesorhabditis, the anchor cell has no apparent role in inducing vulva formation (Sommer and Sternberg, 1994). The vulva precursor cells and the gonad primordium are in the center of the animal at hatching. The vulva precursors then migrate posteriorly in L2 and form a posterior vulva (Sommer and Sternberg, 1994). During this time, the anchor cell becomes the most posterior cell in the gonad primordium shortly after its birth (Figs 2, 5A). It then pioneers the elongation of the gonad towards the posterior vulva (Fig. 5B). If the anchor cell is ablated during L3, when it has just started migrating posteriorly, the gonad stops elongating and the uterus forms in the center of the animal (5/5 animals, Fig. 5C). This experiment demonstrates a new role for the anchor cell: it drives gonad elongation towards the posterior vulva, which results in the specific morphology of the reproductive system in this species.

\section{The ventral uterine precursors and the anchor cell: lineal origin and position}

In Caenorhabditis elegans, the precursors of the ventral uterus are aligned at their birth: Z1.ppa, Z1.ppp, Z4.aaa and Z4.aap from anterior-left to posterior-right. All four cells are competent at an early stage to become the anchor cell (Seydoux et al., 1990), and may become anchor cells in some reductionof-function mutants of C. elegans lin-12 (Sternberg and Horvitz, 1989; P. W. S. and A. P. Newman, unpublished). In normal development, only the central cells may become the single anchor cell, and the specification is unbiased between the two: Z1.ppp becomes the anchor cell in half of the animals, Z4.aaa in the other half. This leads to two asymmetric configurations in the somatic gonad primordium in late L2: if Z1.ppp is the anchor cell (AC), there are two ventral uterine precursors (VUs) anterior to AC and one posterior; if Z4.aaa is the $\mathrm{AC}$, there are two posterior and one anterior VUs. The lineal origin of each $\mathrm{VU}$ is fixed for a given configuration: for instance if Z1.ppp is the $\mathrm{AC}$, the right-anterior $\mathrm{VU}$ is $\mathrm{Z} 4$.aaa (Fig. 6, top panel). After two more rounds of divisions of the VUs in L3, the symmetry around the AC is restored (Kimble, 1981; Newman et al., 1995).

In all monodelphic species studied, the uterus is formed 


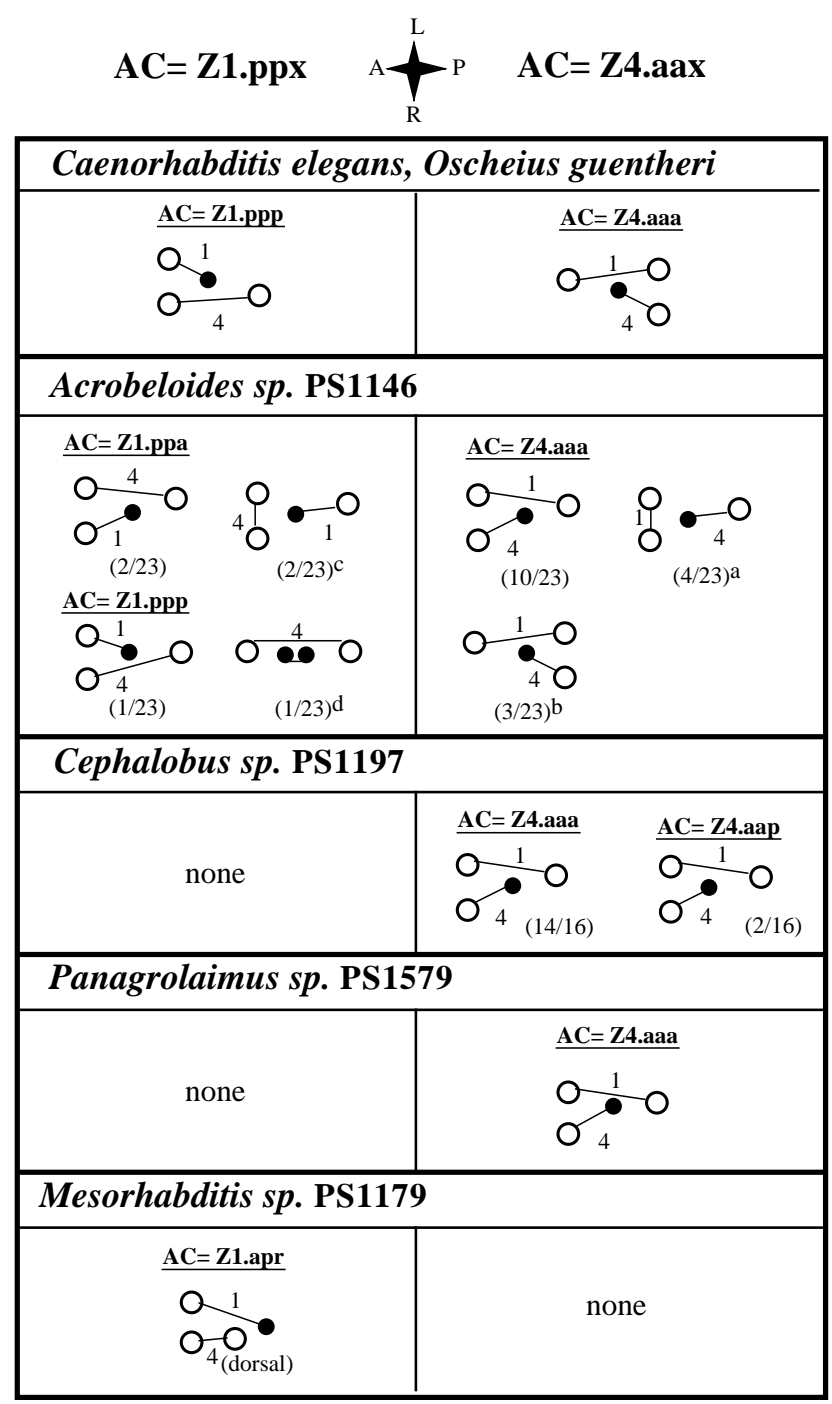

Fig. 6. Lineal origin and configuration in the ventral uterine primordium. Position of nuclei in the ventral uterus at the somatic primordium stage, for animals in which the anchor cell is a descendant of Z1 (left column) or of Z4 (right column). Black, AC; white, VU. The lineal origin of the cells is represented by the lines joining the two daughters of Z1.pp ('1') and of Z4.aa ('4'). For the frequency of each configuration in C. elegans and $O$. guentheri, see the text. For Acrobeloides sp. PS1146 and Cephalobus sp. PS1197, the number of animals in which cell lineage were followed is indicated below each corresponding configuration. ${ }^{\text {a }}$ The anterior left VU was born Z1.ppp in 2/4 animals, Z1.ppa in 1/4, and Z1.ppl in $1 / 4$. In one of them, Z1.pp repeated the lineage of its mother so that the anterior VU was Z1.ppva, and the posterior left VU was Z1.ppvp.

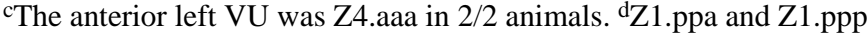
appeared fused in this animal.

from both Z1 and Z4 progeny, and Z1.pap and Z4.apa are the dorsal uterine precursors. However, in the ventral uterus primordium (typically the four daughters of Z1.pp and Z4.aa), variations occur in the lineal origin of the anchor cell (Fig. 2) and in the position of the three VUs relative to the AC (Fig. 6).

By continuous observation of Oscheius guentheri development throughout L2, we found that in 10/11 animals, Z4.aaa became the AC and the configuration with two posterior VUs developed. In one animal, Z1.ppp became the AC and the reverse configuration occurred. By examining anatomy in early L3, we found correspondingly that 92/100 animals showed the configuration with two posterior VUs, $8 / 100$ the configuration with two anterior VUs. In the closely related didelphic Oscheius sp. PS1131, the configuration with two posterior VUs occurred in 43/100 animals. We conclude that, whereas the outcome is unbiased in the didelphic species, in the monodelphic Oscheius guentheri, there is a bias towards Z4.aaa becoming the AC.

In Acrobeloides sp. PS1146, we also find a bias: Z4.aaa became the AC in 17/23 animals that we followed (from the division of Z4.aa and Z1.pp throughout the L3 period until division of the VUs), and Z1.ppa or Z1.ppp became the AC in the remaining $6 / 23$ animals. The final configuration forms slowly during L3 and has either two anterior and one posterior VUs, or one anterior VU and two posterior, with a strong bias against the latter (3/23). But in this species, lineage history does not correlate with final configuration, and there are a range of outcomes (Fig. 6; see legend for details).

In Cephalobus sp. PS1197, the AC is always a descendant of Z4 (16/16 animals), and the final configuration is fixed to two anterior and one posterior VUs (100/100 animals). The lineal origin of the different VUs is also essentially fixed: Z4.aap migrates anterior to Z4.aaa just after its birth, and becomes the anterior right VU, Z1.ppa is anterior left, Z1.ppp is posterior (14/16 animals). In 2/16 animals, the axis of Z4.aa division was reversed, so that the posterior daughter directly became the $\mathrm{AC}$, eliminating the need for movements to reach the final configuration.

Acrobeloides sp. PS1146 and Cephalobus sp. PS1197 are closer to each other than to the other species under investigation (Fig. 1). Interestingly, the most common lineage and configuration in the ventral uterus in PS1146 (10/23 animals, Fig. $6)$ is the one that is fixed in PS1197. Also, whereas in Cephalobus sp. PS1197, Z4.aa always divides before Z1.pp and with a stereotypic geometry (44/44 animals), in Acrobeloides sp. PS1146, this is only a bias: Z4.aa divides more often before (35/55 animals; $27 / 35$ more than 10 minutes before) than with (6/55), or after Z1.pp (14/55; 7/14 more then 10 minutes after). The axis of their division in PS1146 can be antero-posterior as in PS1197, but also left-right, dorso-ventral or oblique (especially for Z1.pp). At birth, Z1.ppp can be in PS1146, as in PS1197, on the left of Z4.aaa (44/60), but also on its right $(8 / 60)$ or anterior (8/60) to it. The first cell to divide appears more likely to become the AC in Acrobeloides $s p$. PS1146: in 17/17 cases where the AC was Z4.aaa, Z4.aa had divided first or with Z1.pp; in 4/6 cases where the AC was a Z1.pp descendant, Z1.pp had divided first (in one of two other animals, it had divided less than 10 minutes later, the other was the aberrant animal (d) in Fig. 6). Thus for the relative timing and orientation of Z1.pp and Z4.aa divisions, for the lineal identity of the AC, and for the lineal history and final configuration of the VUs around the AC, there is a biased variability in Acrobeloides sp. PS1146. The most common outcome in Acrobeloides sp. PS1146 is fixed in Cephalobus sp. PS1197.

In Panagrolaimus sp. PS1579, as in Panagrellus redivivus (Sternberg and Horvitz, 1981), the AC is always Z4.aaa, and the final configuration and lineal origin of the VUs are the same as in Cephalobus sp. PS1197. Z4.aa is anterior to the Z1.pp 
daughters when it divides (in contrast to Cephalobus $s p$. PS1197) and the final configuration is achieved, as in Panagrellus redivivus, by a rapid anterior migration of $\mathrm{Z} 4$.aaa (the AC) in between Z4.aap and Z1.ppa. Note that the single posterior VU, Z1.ppp, has a specific fate in Panagrellus redivivus (Sternberg and Horvitz, 1981).

In Mesorhabditis, as described above, the configuration is extremely asymmetric, with all three VUs anterior to the AC. The AC is always a descendant of Z1 (Figs 2, 6; note the inversion in the orientation of $\mathrm{Z} 1$ division, similar to that described for Panagrellus redivivus males in Sternberg and Horvitz, 1981).

\section{Symmetry breakage in the cell interactions within the ventral uterus}

The specification of the anchor cell is thus biased or fixed in distinct monodelphic lines to either a $\mathrm{Z} 1$ or a $\mathrm{Z} 4$ descendant. This implies that the symmetry of the reciprocal lateral inhibitory interaction between Z1.ppp and Z4.aaa (leading to the choice of AC or VU fates, Fig. 7A) is broken. A highly reproducible lateral inhibitory interaction between these cells could remain in those species where the fates in the ventral uterus are fully fixed (i.e. in species in which Z4.aaa is always the AC, Z4.aaa could reproducibly inhibit the AC fate in

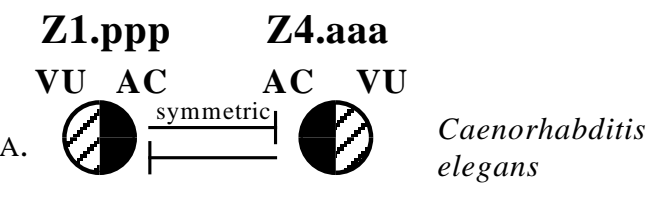

B. $13 \begin{aligned} & \text { Acrobeloides } \\ & \text { PS } 1146\end{aligned}$
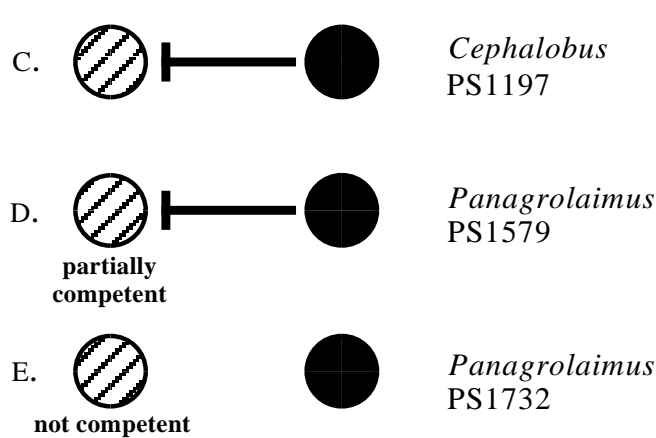

Fig. 7. Cell interactions specifying the anchor cell (AC) and the ventral uterine precursor (VU). Z1.ppp and Z4.aaa are depicted by either black or hatched areas, representing the proportion of animals in which each cell becomes the AC or a VU, respectively. Between them are indicated the extent and direction of lateral signalling, as revealed by lineage and ablation studies. Lateral inhibition is symmetric in C. elegans, asymmetric in Acrobeloides (and in Oscheius guentheri), and unidirectional in Cephalobus. Z1.ppp is only partially competent to become an AC in Panagrolaimus sp. PS1579 (it induces the vulva like the AC but divides like a VU). Z1.ppp is not competent in Panagrolaimus sp. PS1732. Only the final outcome of the interaction is considered, not the initial presence of the ligand or the receptor. For simplicity, Z1.ppa and Z4.aap are not represented.
Z1.ppp; Fig. 7C). Alternatively, the two fates could be exclusively determined by lineage history, or by other signals from outside the ventral uterus (Fig. 7E). To test this, we ablated Z4.aaa (or its mother Z4.aa) in three species (Cephalobus sp. PS1197, Panagrolaimus spp. PS1579 and 1732) in which Z4.aaa (or Z4.aap in PS1197) always becomes the AC, and looked for the presence of an AC (recognizable by its specific morphology, and its cell cycle arrest) and for the extent of vulval induction. The vulval lineages are identical in these three species to that of Panagrellus redivivus (Sternberg and Horvitz, 1982): the vulva is formed from the four precursors P5.p, P6.p, P7.p and P8.p (from anterior to posterior), each of which first divides twice. The four central granddaughters then divide once more transversely; this last round of division is induced by the AC after the P6.p and P7.p divide (in the species considered in Tables 2 and 3). It constitutes a specific fate of P6.pp and P7.pa (M.-A. F. and P. W. S., unpublished).

In Cephalobus sp. PS1197, if the whole gonad is ablated in early L1 larvae, or the AC is ablated in mid-L3, only this last round of division is eliminated (Table 2). If $\mathrm{Z} 4$ is ablated, an $\mathrm{AC}$ forms from the $\mathrm{Z1}$ lineage, and the last round of vulval divisions occurs. Induction also occurs if we ablate Z4.aaa and Z4.aap in L2 (right after their birth), but not if Z1.pp is also ablated. Therefore, Z1.ppa and/or Z1.ppp can replace the anchor cell for vulva induction. A morphologically distinguishable AC is seen, and it does not divide (Table 4). Thus, in Cephalobus PS1197, despite the fact that Z4.aaa normally always becomes the AC, it can be replaced by Z1.ppp (or possibly Z1.ppa) if it is ablated. This implies that Z4.aaa inhibits the AC fate in Z1.ppp in a highly reproducible manner (Fig. 7C).

In Panagrolaimus sp. PS1579, the results are similar for the replacement of Z4.aaa for vulva induction: if Z4.aaa is ablated

Table 2. AC/VU regulation in Cephalobus sp. PS1197: assay of vulva induction

\begin{tabular}{lcccccc}
\hline & & \multicolumn{4}{c}{ Vulva lineages } \\
\cline { 3 - 5 } Cell(s) ablated & Time of ablation & P5.p & P6.p & P7.p & P8.p & No. \\
\hline- & - & UUUU & UUTT & TTUU & UUUU \\
Gonad & Early L1 & UUUU & UUUU & UUUU & UUUU & $8 / 8$ \\
Z1 & Early L1 & UUUU & UUTT & TTUU & UUUU & $2 / 6$ \\
& & UUTT & TTUU & UUUU & UUUU & $2 / 6$ \\
& & UUUU & TTTT & UUUU & UUUU & $1 / 6$ \\
& & UUUU & UTTT & TTUU & UUUU & $1 / 6$ \\
Z4 & \multirow{2}{*}{ Early L1 } & UUUU & UUTT & TTUU & UUUU & $4 / 8$ \\
& & UUUU & TTTT & UUUU & UUUU & $2 / 8$ \\
& & UUUU & UUTT & TTUU & UU U & $1 / 8$ \\
& & UUUU & TTTU & UUUU & UUUU & $1 / 8$ \\
Z1.pp + Z4.aaa & L2 lethargus & UUUU & UUUU & UUUU & UUUU & $5 / 5$ \\
+ Z4.aap & & & & & & \\
Z4.aaa + Z4.aap & L2 lethargus & UUUU & UUTT & TTUU & UUUU & $7 / 9$ \\
& & UUUU & UUTT & OTUU & UUUU & $1 / 9$ \\
& & UUUU & UUTT & TTTU & UUUU & $1 / 9$ \\
AC & \multirow{2}{*}{ Mid-L3 } & UUUU & UUUU & UUUU & UUUU & $6 / 6$
\end{tabular}

The vulva is formed by the progeny of P5.p to P8.p, which first all divide twice in late L3, even in the absence of the gonad. The letters indicate whether and in which orientation their four granddaughters further divided. The nomenclature follows Sternberg and Horvitz, 1986: U, undivided; T, transverse division; $\mathrm{O}$, oblique division. Bold indicates the fate induced by the AC. 
Table 3. AC/VU regulation in Panagrolaimus spp. PS1579 and PS1732: assay of vulva induction

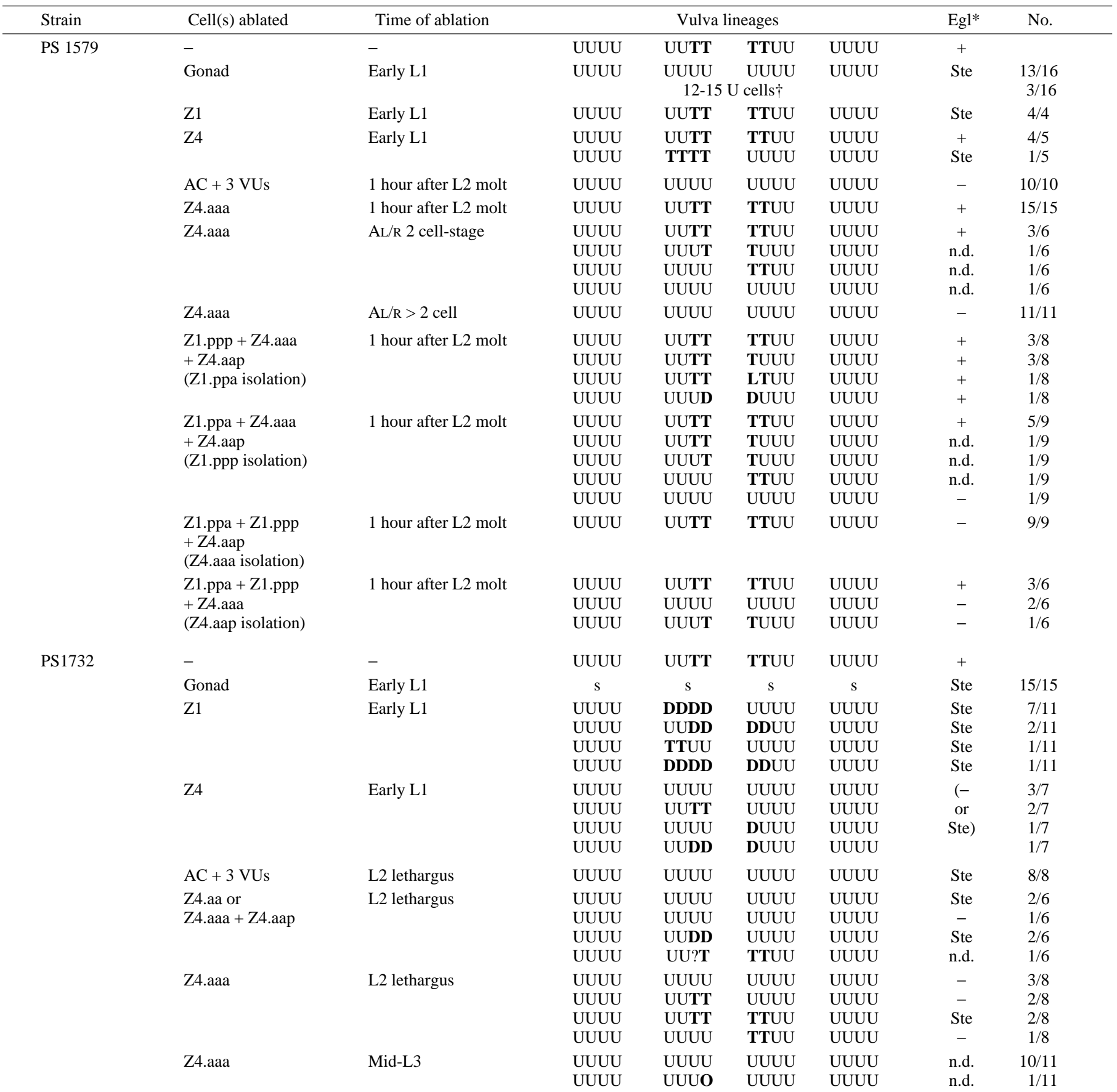

Nomenclature as in Table 2, and $\mathrm{L}=$ longitudinal division, $\mathrm{D}=$ divided, orientation unknown, $\mathrm{s}=$ fused to the epidermal syncytium (non-vulval fate).

*The formation of a functional vulva was scored (column 'Egl') as the ability of nonsterile animals to lay eggs. Ste, sterile.

The L2 molt is defined as the molt between the L2 and the L3 stages.

$\dagger$ In these animals, only 12-15 descendants of $\mathrm{P}(5-8)$.p could be found, presumably because $\mathrm{P}(5-8)$. $\mathrm{p}$ did not all divide twice.

In Panagrolaimus sp. PS1579, only the last transverse round of division of the vulva lineages (in bold) is induced by the AC.

In PS1732, the first two rounds of vulva divisions are induced by Z1 and Z4 in L2, the last one (in bold) by the AC in late L3 (M.-A. F. and P. W. S., unpublished).

Gonad development in PS1732 is similar to that in PS1579, except that Z4 divides earlier (but still after Z1). Note the abnormal centering of the vulva on P6.p upon Z1 ablation, presumably the result of a more anterior position of Z4.aaa (the AC) in the gonad primordium.

and all three VUs are present, vulva induction is always normal, but if all four are ablated, vulval induction is disrupted. Regulation can occur until mid-L3, once the anterior ovary precursors have divided a second time (Table 3). One of the VUs takes on the granular appearance of an $\mathrm{AC}$ and extends to contact the vulva precursor cells (Fig. 8A), but subsequently divides like a normal VU (Fig. 8B-D). Therefore the VUs are still competent to induce the vulva if the normal $\mathrm{AC}$ is ablated, but they cannot arrest their cell cycle, another aspect of the AC fate, and then form a ventral uterus functional in egg-laying 
Table 4. AC/VU regulation in monodelphic species: a summary

\begin{tabular}{lccc}
\hline & \multicolumn{3}{c}{ Replacement of the AC for: } \\
\cline { 2 - 4 } Species & $\begin{array}{c}\text { Vulva } \\
\text { induction }\end{array}$ & Morphology & $\begin{array}{c}\text { Cell cycle } \\
\text { arrest }\end{array}$ \\
\hline Cephalobus sp. PS1197 & + & + & + \\
Panagrolaimus sp. PS1579 & + & + & - \\
Panagrolaimus sp. PS1732 & - or partial & - & - \\
Panagrellus redivivus PS1163 & NA & - & -
\end{tabular}

This table summarizes which aspects of the anchor cell fate are replaced by ventral uterine precursors in different species if Z4.aaa (i.e. the normal AC) is ablated. + indicates replacement, - indicates no replacement. An AC morphology is scored as a cell with a granular cytoplasm that stretches in between the vulva precursor cells in the ventral cord. In Panagrellus redivivus, upon ablation of $\mathrm{Z} 4$.aaa, no $\mathrm{AC}$ is seen (Sternberg and Horvitz, 1982 and M.-A. F., unpublished). NA, non applicable; we find that the AC is not required for vulva induction in $P$. redivivus PS1163 (M.-A. F. and P. W. $\mathrm{S}$, unpublished).

(Tables 3, 4). The two cell fates are thus separable but not mutually exclusive. Any of the three VUs can replace the AC: when Z4.aaa was ablated shortly after birth, before its migration in between Z4.aap and Z1.ppa (see above and Sternberg and Horvitz, 1981), the replacing VU (determined by its granular aspect and its contacting the vulva precursor cells) was Z1.ppa in 3/6 animals, Z1.ppa then Z1.pppa in $1 / 6$, Z4.aap in $2 / 6$. When Z4.aaa was ablated slightly later, after it migrated posteriorly to Z4.aap and Z1.ppa, the replacing cell was Z1.ppp in 4/6 animals, and Z1.pppa in 2/6. Thus Z1.ppa and Z4.aap initially appear to be more competent than Z1.ppp to replace the $\mathrm{AC}$, but they lose competence earlier, presumably because Z4.aaa inhibits them from adopting the AC fate during its migration. Also, each of the three VUs can induce the vulva when isolated from the others, although less fully than Z4.aaa itself (Table 3).

In Panagrolaimus sp. PS1732, all divisions of the vulva precursor cells are induced by the gonad (Table 3, bottom part). The first two rounds of divisions are induced in early L 2 by the somatic gonad (then composed of Z1 and Z4), and the last
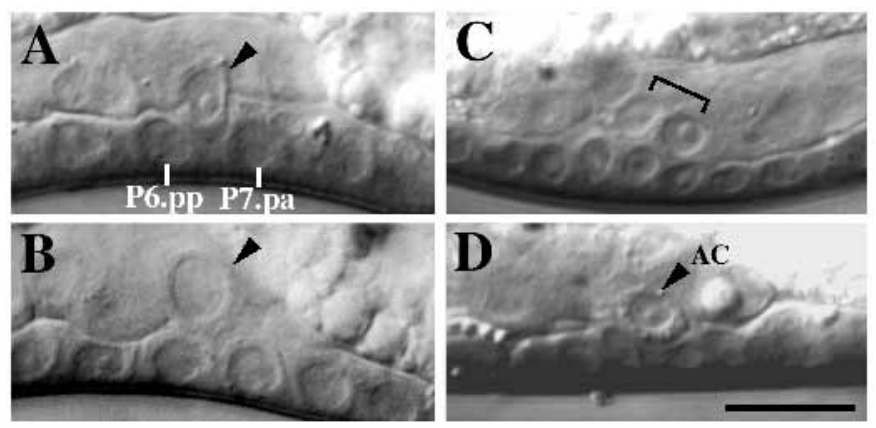

Fig. 8. Division of the ventral uterine precursor replacing the anchor cell upon ablation in Panagrolaimus sp. PS1579. (A-C) AC ablation in early L3. (A) The replacing VU (arrowhead) acquires the granular cytoplasm characteristic of an anchor cell and contacts the vulval precursors P6.pp and P7.pa. (B) It enters prophase. (C) Its daughters then contact the daughters of P6.pp and P7.pa. (D) No ablation, same stage as (C), the AC stays undivided. A dying cell from the postvulval sac lineage is visible on the right. Lateral views, anterior is to the left in A, B, D, to the right in C. Bar: $10 \mu \mathrm{m}$. transverse round by the $\mathrm{AC}$ signalling to P6.pp and P7.pa in late L3 (M.-A. F. and P. W. S., unpublished). Either Z1 or Z4 is sufficient for the first induction, but $\mathrm{Z} 1$ descendants cannot or can only partially take over the inductive role of the $\mathrm{AC}$ in the second induction (Table 3 ). In no case did we see an AC (as judged by cell morphology or cell cycle arrest). The VUs are thus not competent to replace the $\mathrm{AC}$, even for vulva induction (Table 4). In this species, the distinct AC and VU fates of Z4.aaa and Z1.ppa or Z1.ppp are not determined by detectable intercellular signalling between them, but by other asymmetries between the $\mathrm{Z} 1$ and the $\mathrm{Z} 4$ lineages.

\section{DISCUSSION}

\section{Asymmetric gonad development: a novel fate for $\mathbf{Z 4}$}

We show here that the asymmetry of female/hermaphrodite one-armed gonads is reflected at the cellular level in the asymmetry of development of the somatic gonad precursors $\mathrm{Z} 1$ and Z4. Mainly, the development of Z4 progeny is altered. Major differences between $\mathrm{Z1}$ and $\mathrm{Z} 4$ include (1) their different developmental timing, (2) the asymmetric signalling from Z1 to Z4 in Cephalobus sp. PS1197, (3) the differential activities of the anterior and posterior distal tip cells in Oscheius guentheri and Acrobeloides sp. PS1146, (4) the specific cell death of the presumptive posterior distal tip cell in all species but $O$. guentheri and of the presumptive posterior ovary precursors in Mesorhabditis sp. PS1179, and (5) the difference in AC/VU potential between the Z1 and Z4 lineages.

A developmental difference between $\mathrm{Z1}$ and $\mathrm{Z} 4$ in monodelphic species could result from their relative positions along the antero-posterior axis of the animal (for instance if they received different cues from Hox gene patterning), or from their distinct origins in the embryonic lineage. $\mathrm{Z1}$ and $\mathrm{Z} 4$ have the same lineal origin in Turbatrix aceti (Family Panagrolaimidae, related to Panagrolaimus and Panagrellus spp.) as in C. elegans (Sulston et al., 1983). We note that in C. elegans, the sister cells of $\mathrm{Z1}$ and $\mathrm{Z4}$ have different fates: the sister of $\mathrm{Z} 1$ dies and the sister of Z4 becomes the head mesodermal cell. Although male gonads in the order Rhabditida have a single anterior arm, Z1 and Z4 develop symmetrically in Caenorhabditis elegans males (Kimble and Hirsh, 1979). Many other features of male gonad development also differ from monodelphic females/hermaphrodites, for instance the absence of cell death, both in Caenorhabditis elegans and in Panagrellus redivivus (Kimble and Hirsh, 1979; Sternberg and Horvitz, 1981).

It is not clear how often the symmetry between $\mathrm{Z} 1$ and $\mathrm{Z} 4$ fates is broken or restored in nematode evolution. Phylogenetic relations between species should become more precise when molecular data are added to the conventional morphological criteria. It should then be possible to determine the relative frequency of evolution between didelphy and monodelphy. We know of no sure case where didelphy evolved from monodelphy, but it is clear that monodelphy (hence asymmetry of Z1 and Z4 fates) is derived in Oscheius guentheri and very likely derived in Mesorhabditis, and that these species are in distinct monodelphic evolutionary lines, independent from the Cephalobidae and Panagrolaimidae (Fig. 1).

Frequency of change in either direction is a function both of the genetic ease of the change, and of the action of selection. Concerning the latter, we do not know the selective values of 
didelphy or monodelphy (e.g. Sudhaus and Hooper, 1994). Concerning the former, it is possible that a distinction between $\mathrm{Z} 1$ and Z4 could evolve readily because of their distinct origins in the embryonic lineage. We would expect, however, that the acquisition of a novel program for $\mathrm{Z} 4$ requires more, or rarer, mutational steps than restoring the symmetry between $Z 1$ and $\mathrm{Z4}$, because it involves several apparently unrelated downstream events: a change in the timing of the divisions relative to $\mathrm{Z1}$ and its progeny, the activation of cell death in Z4.pp, a novel division and differentiation program for the precursors of the post-vulval sac, a bias in the specification of the AC, etc. Although some of these events may be related mechanistically, we believe that several steps are required. Conversely, evolution from monodelphy to didelphy might only require a single step restoring the initial symmetry between $\mathrm{Z1}$ and Z4. Therefore, didelphy would evolve more readily from a monodelphic species than monodelphy from a didelphic species. Similarly, it appears genetically easier in arthropods to lose than to gain segment diversity (Lewis, 1978), or in plants to evolve a symmetric flower from an asymmetric one, than conversely (Coen and Nugent, 1994).

In $C$. elegans, no monodelphic mutants have yet been isolated. The only gene that can be mutated to cause development of a single gonadal arm (or none at all) is lin-17, which has a defect in the asymmetry of the first division of Z1 and Z4, but does not affect differently these two cells (Sternberg and Horvitz, 1988). A number of $C$. elegans mutants have a distal tip cell migration defect that is more penetrant in the posterior arm (Hedgecock et al., 1990). The initial symmetry between Z1 and Z4 is probably not affected, but this suggests that, in a didelphic species, the two arms might already be asymmetric in their late development, by growing and turning in a different environment along the antero-posterior axis of the animal. Monodelphy could originate with a practically irreversible alteration of late posterior arm development, followed in evolution by an earlier breakage of symmetry between Z1 and Z4. Note that in Cephalobus sp. PS1197, posterior arms induced by ablations show a migration defect, which could have evolved after the reduction of the posterior arm, or might be a cause for the reduction. Asymmetry could in this case arise through the action of deleterious mutations in the context of the slight preexisting asymmetries in which the anterior and posterior arms develop along the antero-posterior axis of the animal. This asymmetry would be followed by the selection of the progressive reduction of the already dysfunctional posterior arm.

Oscheius guentheri, because it appears to be the result of such a recent evolutionary reduction of the posterior arm, is particularly useful to study the evolution of monodelphy (Sudhaus and Hooper, 1994). Gonads that are only slightly asymmetric, with germ line in both arms, and highly variable development of the reduced arm, are also found in other genera comprising both didelphic and monodelphic species, such as Pratylenchus (Dickerson, 1962; Roman and Hirschmann, 1969) or Xiphinema (in which the anterior arm is reduced) (Luc, 1981). On the other hand, species from older stable monodelphic evolutionary lines seem to use larger asymmetries earlier in development and more specific mechanisms, like programmed cell death, to execute the breakage of symmetry.

\section{Elimination of cells}

The distal tip cells are essential in shaping the ovary arms of the gonad: ablation of one of them in C. elegans prevents the corresponding arm from growing (Kimble and White, 1981). In most of the monodelphic species analyzed, we find that the putative posterior distal tip cell is eliminated by programmed cell death. The correlation between cell death and morphology is particularly striking in Mesorhabditis sp. PS1179, where the posterior ovary precursors also die, thereby eliminating any structure posterior to the uterus. In this species, the presumptive posterior distal tip cell is never born: Z4.p dies. In Panagrolaimus spp., Z1.aa is born much earlier than Z4.pp (and thus can act earlier on the germ line) and death of Z4.pp occurs shortly after its birth: these two mechanisms are likely to prevent Z4.pp from acting as a distal tip cell. In Acrobeloides sp. PS1146 and Cephalobus sp. PS1197, Z4.pp dies very late, at the molt to the L4 stage. At this point, the anterior arm is already much more developed than the posterior arm. Therefore differential activity of Z1.aa and Z4.pp, not cell death, appears to be the primary developmental asymmetry involved in shaping the gonad in these species.

Programmed cell death occurs in a variety of developmental contexts: in animals undergoing metamorphosis, during modeling of specific tissues like the digits of the vertebrate limbs, in eliminating supernumerary cells in the nervous system, or vestigial structures like the pro- and mesonephros, aortic arches, or the tail in humans (Oppenheim, 1991; White, 1995). The latter cases are analogous to the occurrence of cell death in the posterior ovary in monodelphic nematodes. Many cells die during nematode development (Sommer and Sternberg, 1996; Sternberg and Horvitz, 1981; Sulston and Horvitz, 1977; Sulston et al., 1983). In C. elegans mutants in which cell death is prevented, there is an excess of cells but no major morphological change, and the worms develop normally (Ellis and Horvitz, 1986). In the monodelphic species examined here, there is a correlation between changes in cell death and changes in the morphology of an organ. Evolutionarily, the question is whether the regulation of cell death can be the primary event in morphological changes, or whether it only serves to eliminate cells rendered unnecessary by prior modifications. Interestingly, the only species where the presumptive posterior distal tip cell does not die is Oscheius guentheri, where the loss of the posterior arm appears to be a recent evolutionary event (Sudhaus and Hooper, 1994). Thus, at least in the phylogenetic line leading to O. guentheri, elimination of the distal tip cell by programmed cell death is not the primary event leading to monodelphy. Rather, it appears as a feature of stable, relatively old monodelphic groups.

\section{Evolution of cell roles}

The program downstream of somatic primordium fate specification can evolve. For example, the posterior ovary precursors divide to a greater or lesser extent in different species and differentiate to form a post-vulval sac of varying size. Evolution of a cell program is however particularly spectacular for a cell that does not divide further, the anchor cell. In Mesorhabditis sp. PS1179, the anchor cell has lost its role in inducing and patterning the vulva, as the vulva apparently forms autonomously in the posterior part of the animal (Sommer and Sternberg, 1994). However, the AC has a new posterior position in the ventral uterine primordium and a new role in driving elongation of the gonad towards the posterior vulva. This role resembles that of the linker cell in development of 
the one-armed male gonad (Kimble and Hirsh, 1979; Sternberg and Horvitz, 1981), except that the anchor cell specifically contacts the developing vulva, and does not reach the proctodeum (in mutant males with vulval tissue, the linker cell contacts the vulva and fails to reach the proctodeum, Sulston and Horvitz, 1981). This might be a case where genetic information was transferred between the two sexes by rewiring of the sex determination program with its downstream events.

\section{Evolution of cell interactions}

No signalling is known to occur between $\mathrm{Z} 1$ and $\mathrm{Z} 4$ in $C$. elegans, only between their central descendants where they intermingle to form the uterus. There, the interaction between Z1.ppp and Z4.aaa is symmetric and determines which of the two will become the single anchor cell (Kimble, 1981). We find that in all monodelphic species, asymmetric signalling occurs between the $\mathrm{Z} 1$ and $\mathrm{Z} 4$ lineages, either between $\mathrm{Z} 1$ and Z4 themselves early on (in Cephalobus sp. PS1197), or between their descendants in the ventral uterus. In the first case, a novel asymmetric interaction appears; in the second, the symmetry of existing interactions is broken.

Asymmetries between the Z1 and Z4 lineages can cause their descendants of the ventral uterus to adopt a directional pattern of cell interactions identical from animal to animal (Cephalobus sp. PS1197; Fig. 7C). Early asymmetric signalling between Z1 and Z4 in Cephalobus sp. PS1197 is followed at a later stage by a reciprocal asymmetric interaction between their descendants Z4.aax and Z1.ppx. This initial asymmetry establishes a cascade of asymmetric interactions across cellular generations in parallel lineages.

Moreover, intercellular signalling in the AC/VU group apparently disappears altogether in some species, and the cells of the ventral uterus acquire their distinct fates independently from each other in Panagrolaimus sp. PS1732. This is analogous to variations in the mechanisms of mesoderm specification in the 4D blastomere in mollusks (Freeman and Lundelius, 1992; van den Biggelaar, 1996; van den Biggelaar and Guerrier, 1979). Although there is no interaction between the Z1.pp and the Z4.aa progeny, some other asymmetric extracellular signal might nonetheless specify which of the cells becomes the $\mathrm{AC}$ (this would imply that asymmetry in the positioning of $\mathrm{Z} 1$ and $\mathrm{Z} 4$ progeny through lineage is important).

Surprisingly, we find all intermediate types of specification: biased outcome (Fig. 7B) and partial loss of competence (Fig. 7D). Biased outcome is found in C. elegans for the specification of the P11 and P12 cells in the posterior ventral cord (Sulston and Horvitz, 1977). We speculate that biased outcome or partial loss of competence represent evolutionary intermediates.

The early differentiation between $\mathrm{Z} 1$ and $\mathrm{Z} 4$ could be executed at the molecular level by differential regulation in their descendants either of a signalling molecule for the interaction, upstream of the actual specification program (biased or directional cell interactions), or of some molecules directly acting in autonomous specification. A similar molecular pathway can be used in Caenorhabditis and Drosophila development for distinct modes of cell interactions: symmetric interactions with a stochastic, unpredictable outcome; biased interactions; reproducible, directional interactions between unrelated cells, or between sisters (Artavanis-Tsakonas et al., 1995; Sternberg, 1991). Here we see, for one specific cell interaction, an evolutionary transformation between these modes.

At the molecular level, lateral signalling between Z1.ppp and Z4.aaa in C. elegans involves the receptor LIN-12 (similar to Drosophila Notch; Greenwald et al., 1983; Yochem et al., 1988) and its transmembrane Delta-like ligand LAG-2 (Henderson et al., 1994; Tax et al., 1994). Both receptor and ligand are initially expressed symmetrically in Z1.ppp and Z4.aaa. Then, a seemingly stochastic difference between the two cells is reinforced by differential signalling between the cells, and positive feedback loops of expression of the receptor (and probably its ligand) within the cells. The final outcome is a stable state where the anchor cell (Z1.ppp in half of the animals, Z4.aaa in the other half) expresses LAG-2. This activates the LIN-12 receptor expressed by the ventral uterine cell (Z4.aaa or Z1.ppp, respectively) (Seydoux and Greenwald, 1989; Wilkinson et al., 1994). The anchor cell is the default state.

In monodelphic species where Z1.ppp and Z4.aaa interact, if the interaction uses the same molecular pathway as in $C$. elegans, the bias in cell interactions could originate in Z4.aaa constitutively expressing a LAG-2 like ligand at a higher level, or expressing less LIN-12 than its sister on one hand, and their Z1 counterparts on the other. The division of Z4.aa appears asymmetric in Panagrolaimus sp. PS1579, as judged by the size of the daughters' nuclei right after birth (Z4.aaa is smaller than Z4.aap). Such an asymmetric division could differentially segregate determinants of the level of LIN-12 or LAG-2 expression, as do asymmetric divisions of neuron precursors in the ferret cerebral cortex for Notch1 (Chenn and McConnell, 1995), or of Drosophila sensory organ precursors for Numb and Prospero, acting upstream of Notch (Hirata et al., 1995; Knoblich et al., 1995). On the other hand, differential expression between the Z4.aaa and the Z1 counterparts is likely to come from earlier asymmetries between the $\mathrm{Z} 1$ and $\mathrm{Z} 4$ lineages. Bias in the signalling could also follow if the mothers divide at different times, or in different positions (e.g. the correlation between $\mathrm{AC}$ specification and the relative timing of division of Z1.pp and Z4.aa in Acrobeloides sp. PS1146 presented above), since the initial state of the interaction is likely to be crucial in determining the final outcome.

Specification of the anchor cell is unbiased in C. elegans, in which there is no primary asymmetry between $\mathrm{Z} 1$ and Z4 (in contrast to all monodelphic species examined). Such an indeterminancy is actually extremely rare in C. elegans development: other examples are the respective antero-posterior migration of the left and right $\mathrm{P}$ cells in the ventral cord, and of symmetric left and right progeny of the B cell in the male (Chamberlin and Sternberg, 1993; Sulston and Horvitz, 1977). It appears to be confined to such cases where, although the symmetry needs to be broken in each individual animal, there is no primary asymmetry between the cells ( $\mathrm{Z} 1$ and $\mathrm{Z} 4$ for the $\mathrm{AC} / \mathrm{VU}$ specification), putting a constraint on the possibility of their prior differentiation. Breakage of symmetry in the ventral uterine group is probably not the primary event in the evolution leading to monodelphy, but rather a consequence of the differentiation between $\mathrm{Z} 1$ and $\mathrm{Z} 4$ involved in posterior arm reduction. That this cell interaction becomes asymmetric in all monodelphic species in which the symmetry between $\mathrm{Z} 1$ and $\mathrm{Z} 4$ is broken, demonstrates that stochastic events of cell deter- 
mination are unstable in nematode evolution, leading to reproducible development.

We are very grateful to L. Carta and W. Sudhaus for strains, to P. De Ley for species identifications, and to all those who collected soil samples for the Caltech worm collection. We thank members of the laboratory for discussions, and D. Anderson, T. Clandinin, J. Liu, A. Newman, R. Palmer and R. Sommer for comments on the manuscript. This work was supported by fellowships from the European Molecular Biology Organisation and the Human Frontier Science Program. P.W.S. is an investigator with the Howard Hughes Medical Institute.

\section{REFERENCES}

Akam, M., Averof, M., Castelli-Gair, J., Dawes, R., Falciani, F. and Ferrier, D. (1994). The evolving role of Hox genes in arthropods. Development Supplement 209-215.

Anderson, R. V. and Darling, H. M. (1964). Embryology and reproduction of Ditylenchus destructor Thorne, with emphasis on gonad development. Proc. Helm. Soc. Wash. 31, 240-256.

Andrássy, I. (1976). Evolution as a Basis for the Systematization of Nematodes. 288pp. London: Pitman Publishing Ltd.

Andrássy, I. (1984). Klasse Nematoda. 509pp. Stuttgart: Gustav Fischer Verlag.

Artavanis-Tsakonas, S., Matsuno, K. and Fortini, M. E. (1995). Notch signaling. Science 268, 225-232.

Carroll, S. B. (1994). Developmental regulatory mechanisms in the evolution of insect diversity. Development Suppl., 217-223.

Chamberlin, H. M. and Sternberg, P. W. (1993). Multiple cell interactions are required for fate specification during male spicule development in Caenorhabditis elegans. Development 118, 297-323.

Chenn, A. and McConnell, S. K. (1995). Cleavage orientation and the asymmetric inheritance of Notch1 immunoreactivity in mammalian neurogenesis. Cell 82, 631-641.

Chin, D. A. (1977). Embryonic and post-embryonic development of Cylindrocorpus longistoma (Nematoda: Cylindrocorporidae). Nematologica 23, 62-70.

Chitwood, B. G. and Chitwood, M. B. (1974). Introduction to Nematology. Baltimore: University Park Press.

Coen, E. S. and Nugent, J. M. (1994). Evolution of flowers and inflorescences. Development Supplement 107-116.

Dickerson, O. J. (1962). Gonad development in Pratylenchus crenatus Loof and observations on the female genital structures of $P$. penetrans. Proc. Helm. Soc. Wash. 29, 173-176.

Duboule, D. (1994). Temporal colinearity and the phylotypic progression: a basis for the stability of a vertebrate Bauplan and the evolution of morphologies through heterochrony. Development Supplement 135-142.

Ellis, H. M. and Horvitz, H. R. (1986). Genetic control of programmed cell death in the nematode Caenorhabditis elegans. Cell 44, 817-829.

Epstein, H. F. and Shakes, D. C. (1995). Caenorhabditis elegans: Modern biological analysis of an organism. In Methods in Cell Biology 48. New York: Academic Press.

Freeman, G. and Lundelius, J. W. (1992). Evolutionary implications of the mode of D quadrant specification in coelomates with spiral cleavage. J. evol. Biol. 5, 205-247.

Greenwald, I. S., Sternberg, P. W. and Horvitz, H. R. (1983). The lin-12 locus specifies cell fates in Caenorhabditis elegans. Cell 34, 435-444.

Hedgecock, E. M., Culotti, J. G. and Hall, D. H. (1990). The unc-5, unc-6, and $u n c-40$ genes guide circumferential migrations of pioneer axons and mesodermal cells on the epidermis in C. elegans. Neuron 4, 61-85.

Henderson, S. T., Gao, D., Lambie, E. J. and Kimble, J. (1994). lag-2 may encode a signaling ligand for the GLP-1 and LIN-12 receptors of $C$. elegans. Development 120, 2913-2924.

Hirata, J., Nakagoshi, H., Nabeshima, Y.-I. and Matsuzaki, F. (1995). Asymmetric segregation of the homeodomain protein prospero during Drosophila development. Nature 377, 627-630.

Hirschmann, H. (1962). The life cycle of Ditylenchus triformis (Nematoda: Tylenchida) with emphasis on post-embryonic development. Proc. Helm. Soc. Wash. 29, 30-43.

Hirschmann, H. (1977). Anguina plantaginis n. sp. parasitic on Plantago aristata with a description of its developmental stages. J. Nematol. 9, 229243

Hirschmann, H. and Triantaphyllou, A. C. (1967). Mode of reproduction and development of the reproductive system of Helicotylenchus dihystera. Nematologica 13, 558-574.

Horvitz, H. R. and Sternberg, P. W. (1991). Multiple intercellular signalling systems control the development of the C. elegans vulva. Nature 351, 535541.

Kimble, J. (1981). Lineage alterations after ablation of cells in the somatic gonad of Caenorhabditis elegans. Dev. Biol. 87, 286-300.

Kimble, J. and Hirsh, D. (1979). Post-embryonic cell lineages of the hermaphrodite and male gonads in Caenorhabditis elegans. Dev. Biol. 70 396-417.

Kimble, J. E. and White, J. G. (1981). On the control of germ cell development in Caenorhabditis elegans. Dev. Biol. 81, 208-219.

Knoblich, J. A., Jan, L. Y. and Jan, Y. N. (1995). Asymmetric segregation of Numb and Prospero during cell division. Nature 377, 624-627.

Lewis, E. B. (1978). A gene complex controlling segmentation in Drosophila. Nature 276, 565-570.

Lorenzen, S. (1981). Entwurf eines phylogenetischen Systems der freilebenden Nematoden. In Veröffentlichungen des Instituts für Meeresforschung in Bremerhaven. 472pp.

Luc, M. (1981). Observations on some Xiphinema species with the female anterior genital branch reduced or absent (Nematoda : Longidoridae). Revue Nématol. 4, 157-167.

Maggenti, A. (ed.) (1981). Classification of Nemata. In General Nematology, pp. 305-362. New York: Springer-Verlag.

Newman, A. P., White, J. G. and Sternberg, P. W. (1995). The C. elegans lin12 gene mediates induction of ventral uterine specialization by the anchor cell. Development 121, 263-271.

Oppenheim, R. W. (1991). Cell death during development of the nervous system. Аnпи. Rev. Neurosci. 14, 453-501.

Roman, J. and Hirschmann, H. (1969). Embryogenesis and postembryogenesis in species of Partylenchus (Nematoda: Tylenchidae) Proc. Helm. Soc. Wash. 36, 164-174.

Seydoux, G. and Greenwald, I. (1989). Cell autonomy of lin-12 function in a cell fate decision in C. elegans Cell 57, 1237-1245.

Seydoux, G., Schedl, T. and Greenwald, I. (1990). Cell-cell interactions prevent a potential inductive interaction between soma and germline in $C$. elegans. Cell 61, 939-951.

Slack, J. M. W., Holland, P. W. H. and Graham, C. F. (1993). The zootype and the phylotypic stage. Nature 361, 490-492.

Sommer, R. J., Carta, L. K., Kim, S.-Y. and Sternberg, P. W. (1996). Morphological, genetic and molecular description of Pristionchus pacificus sp. n. (Nematoda, Diplogasteridae). Fund. Appl. Nematol. in press.

Sommer, R. J., Carta, L. K. and Sternberg, P. W. (1994). The evolution of cell lineage in nematodes Development Suppl., 85-95.

Sommer, R. J. and Sternberg, P. W. (1994). Changes of induction and competence during the evolution of vulva development in nematodes. Science 265, 114-118

Sommer, R. J. and Sternberg, P. W. (1996). Apoptosis and change of competence limit the size of the vulva equivalence group in Pristionchus pacificus: a genetic analysis. Curr. Biol. 6, 52-59.

Steiner, G. (1935). Opuscula miscellanea nematologica, II. Proc. Helminth. Soc. Washington 2, 104-110.

Sternberg, P. W. (1991). Control of cell lineage and cell fate during nematode development. Curr. Top. Dev. Biol. 25, 177-224.

Sternberg, P. W. and Horvitz, H. R. (1981). Gonadal cell lineages of the nematode Panegrellus redivivus and implications for evolution by the modification of cell lineage. Dev. Biol. 88, 147-166.

Sternberg, P. W. and Horvitz, H. R. (1982). Postembryonic nongonadal cell lineages of the nematode Panagrellus redivivus: Description and comparison with those of Caenorhabditis elegans. Dev. Biol. 93, 181-205.

Sternberg, P. W. and Horvitz, H. R. (1986). Pattern formation during vulval development in Caenorhabditis elegans. Cell 44, 761-772.

Sternberg, P. W. and Horvitz, H. R. (1988). lin-17 mutations of C. elegans disrupt asymmetric cell divisions. Dev. Biol. 130, 67-73.

Sternberg, P. W. and Horvitz, H. R. (1989). The combined action of two intercellular signalling pathways specifies three cell fates during vulval induction in C. elegans. Cell 58, 679-693.

Sudhaus, W. (1976). Vergleichende Untersuchungen zur Phylogenie, Systematik, Ökologie, Biologie und Ethologie der Rhabditidae (Nematoda). In Zoologica 43

Sudhaus, W. and Hooper, D. J. (1994). Rhabditis (Oscheius) guentheri sp.n., 
an unusual species with reduced posterior ovary, with observations on the Dolichura and Insectivora groups (Nematoda: Rhabditidae). Nematologica 40, 508-533.

Sulston, J. and Horvitz, H. R. (1977). Postembryonic cell lineages of the nematode Caenorhabditis elegans. Dev. Biol. 56, 110-156.

Sulston, J. E. and Horvitz, H. R. (1981). Abnormal cell lineages in mutants of the nematode Caenorhabditis elegans. Dev. Biol. 82, 41-55.

Sulston, J. E., Schierenberg, E., White, J. G. and Thomson, J. N. (1983). The embryonic cell lineage of the nematode Caenorhabditis elegans. Dev. Biol. 100, 64-119.

Tax, F. E., Yeargers, J. J. and Thomas, J. H. (1994). Sequence of C. elegans lag-2 reveals a cell-signalling domain shared with Delta and Serrate of Drosophila. Nature 368, 150-154.

Truskova, G. M. (1971). Novyj vid roda Acrobeloides Nematoda, Cephalobidae. (A new species of the genus Acrobeloides, Nematoda, Cephalobidae). Zool. Zhurn. 50, 434-436.

van den Biggelaar, J. A. M. (1996). Cleavage pattern and mesentoblast formation in Acanthochiton crinitus (Polyplacophora, Mollusca) Dev. Biol. 174, 423-430.

van den Biggelaar, J. A. M. and Guerrier, P. (1979). Dorsoventral polarity and mesentoblast determination as concomitant results of cellular interactions in the mollusk Patella vulgata. Dev. Biol. 68, 462-471.

White, K. (1995). Cell death returns to its roots. Current Biology 5, 371-372.

Wilkinson, H. A., Fitzgerald, K. and Greenwald, I. (1994). Reciprocal changes in expression of the receptor lin-12 and its ligand lag-2 prior to commitment in a C. elegans cell fate decision. Cell 79, 1187-1198.

Wood, W. B. (1988). The Nematode Caenorhabditis elegans. Cold Spring Harbor, New York: Cold Spring Harbor Laboratory.

Yochem, J., Weston, K. and Greenwald, I. (1988). C. elegans lin-12 encodes a transmembrane protein similar to Drosophila Notch and yeast cell cycle gene products. Nature 335, 547-550.

(Accepted 23 April 1996) 